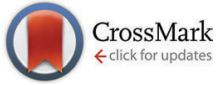

Cite this: Phys. Chem. Chem. Phys., 2014, 16, 27074

Received 14th October 2014 Accepted 30th October 2014 DOI: $10.1039 / c 4 c p 04602 a$ www.rsc.org/pccp

\title{
Depth-selective microscopic observation of a photomobile liquid crystal polymer under UV illumination $\dagger$
}

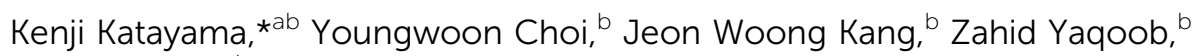 \\ Peter T. C. So, ${ }^{b}$ Tomomi Fujii, ${ }^{a}$ Shota Kuwahara, ${ }^{a}$ Kiyohide Takado ${ }^{c}$ and \\ Tomiki Ikeda*c
}

\begin{abstract}
By using the depth selective imaging method, we studied the UV induced change in a photomobile liquid crystalline polymer film. With $1 \mu \mathrm{m}$ depth resolution, each slice inside the film was selectively observed. A network-like structure mixed with the ordered and disordered regions of molecules in the middle of the film, and a rubbed polymer layer at the bottom of the film were observed. In each slice of the film, the phase change induced by UV light was observed strongly dependent on the director direction, which indicates the ordering change of the liquid crystalline molecules in the director direction. It took several tens of seconds for the ordering change caused by the collaborative interaction between the molecules. Furthermore, it was suggested that the UV induced change travelled from the bottom layer to the middle layer on the micron order.
\end{abstract}

\section{Introduction}

Liquid crystalline polymers have promising properties for various applications due to both the properties of liquid crystals (LCs) and polymers. Molecular alignment can be controlled by stimuli such as electric field, while elasticity is given by polymerization. In cross-linked LC polymers, the orientation of mesogens is strongly coupled with the conformation of the polymer chains, and macroscopic deformation can be induced by heat, electricity and light. ${ }^{1,2}$ When the photochromic molecules are incorporated into the cross-linked LC polymers, the deformation was controlled by light. By introducing azobenzene as a photochromic molecule, the macroscopic motion is controllable by the combination of the UV and visible light, because the trans isomer is photo-isomerized by UV light and it is returned back by a visible light. By coupling the photo-isomerization and the surrounding polymer, not only contraction or expansion, ${ }^{3}$ but also three-dimensional motion

\footnotetext{
${ }^{a}$ Department of Applied Chemistry, Chuo University, 1-13-27, Kasuga, Bunkyo, Tokyo 112-8551, Japan. E-mail: kkata@kc.chuo-u.ac.jp; Fax: +81-3-3817-1913; Tel: $+81-3-3817-1913$

${ }^{b}$ Laser Biomedical Research Center, Massachusetts Institute of Technology, 77, Massachusetts Ave, Cambridge, MA 02139, USA

${ }^{c}$ Research and Development Initiative, Chuo University, 1-13-27, Kasuga, Bunkyo, Tokyo 112-8551, Japan. E-mail: tikeda@tamacc.chuo-u.ac.jp

$\dagger$ Electronic supplementary information (ESI) available: The molecular structure of the monomer and crosslinker, the Raman imaging using the other peaks, the SpeCRPM images for the polarization perpendicular with the director. The movies corresponding to Fig. 3. See DOI: 10.1039/c4cp04602a
}

such as film bending ${ }^{4,5}$ have been demonstrated. Furthermore, a plastic motor, ${ }^{6}$ an inch-size worm, ${ }^{7}$ and a robotic arm $^{8}$ only by using light have been developed.

The overall mechanism for the three-dimensional motions was understood that the surface region of the film was contracted more than the bottom side of it by the excess UV absorption in the surface region. ${ }^{4}$ However, the understanding from the viewpoint of the molecular interactions is still under investigation, although much effort has been made for it. ${ }^{9}$ In the photomobile films in motion, the initial nanometer-scale change due to the photoisomerization should be expanded into the micron- or milli-scale region via the collaborative interactions between molecules, polymer chains, domains. This kind of spatial expansion of the molecular interactions is difficult to understand because both temporal and spatial changes range over many orders. In the previous paper, ${ }^{10}$ we studied the UV-induced dynamics from the viewpoint of the temporal change, using the heterodyne transient grating method, which gives the dynamics from a few nanoseconds to a hundred seconds after a single pulse-light irradiation. ${ }^{11,12}$ Since the refractive index change was detected in this method, it is suitable for LC or polymer samples because they typically have no optical absorption bands in the visible wavelength region. ${ }^{13,14}$ We could observe the initial film contraction on the order of several hundreds of nanoseconds, the subsequent reorientation and molecular rotation dynamics from a few microseconds to a hundred milliseconds, and the return of the cis isomer to the trans isomer on tens of seconds. Furthermore, we figured out that the molecular orientation by the UV irradiation was highly anisotropic under the conditions preferred for the mobility. ${ }^{10}$ 
In this study, we studied the dynamics from the viewpoint of the spatial change. For depth-selective measurements, we used speckle correlation reflection phase microscopy (SpeCRPM), ${ }^{15}$ with a depth resolution of about $1 \mu \mathrm{m}$ and a field of view up to about hundred micron. Phase microscopy is suitable for the observation of the LC polymer, because the structural or orientational change induce the variation in the refractive index. Furthermore, the depth selective observation and the wide field of view of SpeCRPM is beneficial for the LC polymer sample, because it is consisted of different layers with an inhomogeneous structure inside on the order of nano- to micron-scale and the UV induced change could be observed at a video rate. Here we studied the polarization dependent change induced by UV irradiation using SpeCRPM, and also the structure was studied using Raman microscopy.

\section{Experimental}

The general principle and setup of SpeCRPM was described in detail recently, ${ }^{15}$ and a brief outline is given. In this technique, a time-varying speckle-field is used as an illumination source. By constructing Linnik-type configuration, identical speckle-fields are introduced on two arms of the interferometry. The detection port of the microscope is based on the off-axis geometry offering single-shot imaging capability. By using the digital holographic imaging method complex electric field images, amplitude and phase, for objects can be retrieved. When the speckle-field is changing fast enough within a camera acquisition time, a number of independent speckle patterns are averaged out leaving clear interference fringes overlaying object images. The strength of the interference is highly sensitive to the optical path length difference introduced between the two interferometric arms due to the 3-D nature of the speckle-field. The interference quickly decorrelated along the optical axis of the microscope allowing us the confocal equivalent axial resolution, $1.03 \mu \mathrm{m}$. More details about SpeCRPM can be found in ref. 15 .

A mode-locked Ti:sapphire laser was used as a light source, which has a center wavelength of $800 \mathrm{~nm}$ and a spectral bandwidth of $17 \mathrm{~nm}$. The speckle-field was circular polarized on the sample plane, thus each polarization component was selectively measured using a polarizer. The UV light source was a UV curing device (UV75, Thorlabs, $<40 \mathrm{~mW}$ ), which was fixed at the side of the sample, and the irradiation intensity was about $10 \mathrm{~mW} \mathrm{~cm}^{-2}$.

A custom-built Raman NIR confocal Raman microscopy ${ }^{16,17}$ was also used to study the molecular orientation in the film. Briefly, $785 \mathrm{~nm}$ wavelength from Ti:sapphire laser (3900S, Spectra-Physics) was used as an excitation source. High-speed $\mathrm{XY}$ scanning was performed using the galvanometer mirrors (CT-6210, Cambridge Technology). A 1.2 NA water immersion objective lens (UPLSAPO60XWIR $60 \times / 1.20$, Olympus) was used to both focus the laser light onto the sample and to collect the back-scattered light. The axial resolution was around $2 \mu \mathrm{m}$. A linear polarizer was inserted in the beam path to control the polarization. From the film, $100 \times 100$ spectra were acquired from $150 \times 150 \mu \mathrm{m}$ area with $15 \mathrm{~mW}$ power and the integration time was $50 \mathrm{~ms}$ per pixel. The total measurement time was approximately 8.5 minutes.

The sample preparation procedure was described in the previous paper, ${ }^{10}$ and it is briefly explained here. The monomer molecule was 6-[4-(4-hexyloxyphenylazo)phenoxy]hexylacrylate (A6AB6), and 4,4'-bis[6-(acryloyloxy)hexyloxy]azobenzene (DA6AB) was used as a crosslinker (ESI, $\dagger$ Fig. S1). The monomer and crosslinker were solved in chloroform. After that, Irgacure ${ }^{\circledR} 784$ ( $2 \mathrm{~mol} \%$ ) was added as an initiator for polymerization, and chloroform was removed by evaporation. The mixture sample was solved at $110{ }^{\circ} \mathrm{C}$ and put in a LC cell $(15 \times 20 \mathrm{~mm})$, which has an alignment polyimide layer with a thickness of a few microns on the internal side of the cell. The temperature was decreased to $88{ }^{\circ} \mathrm{C}$, and it was polymerized by irradiating a highpressure mercury lamp (USHIO, OPM2-502HQ) with a wavelength $>540 \mathrm{~nm}$ with an intensity of $2 \mathrm{~mW} \mathrm{~cm}^{-2}$ for $2 \mathrm{~h}$. The final thickness of the film was $16 \mu \mathrm{m}$. For the microscopic measurements, only the top glass was peeled off from the LC cell to get a better image. After taking it out from the cell, it was confirmed that it could be bent under UV illumination typically at $10 \mathrm{~mW} \mathrm{~cm}^{-2}$, and returns back to the original position by illumination of a visible light $(>540 \mathrm{~nm})$ typically at $40 \mathrm{~mW} \mathrm{~cm} \mathrm{~cm}^{-2}$.

\section{Results and discussions}

First, the sample was scanned by SpeCRPM in the depth direction, and the typical result is shown in Fig. 1. The sample was imaged from the top surface to the internal slices downward with a step of $1 \mu \mathrm{m}$. There were debris and scratches on the top surface. Further going into the film, we could find a domain-like structure in the region of 5-15 microns typically.
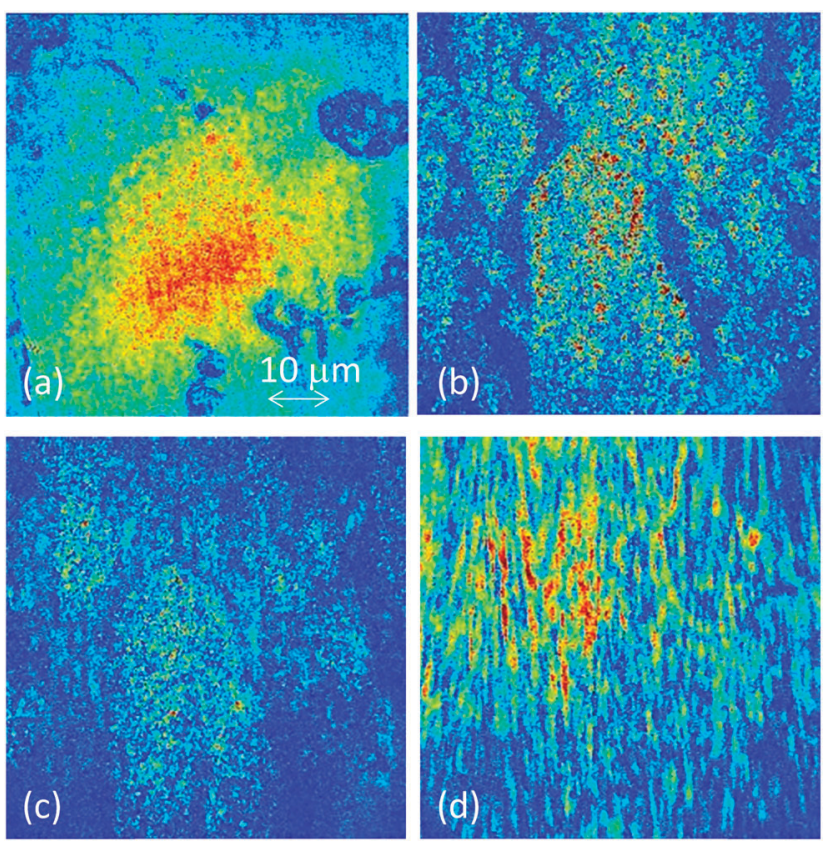

Fig. 1 Depth scan of a photomobile liquid crystalline polymer film by using SpeCRPM. The images shown here correspond to those at 0, 8.0, 12.0 and $18.4 \mu \mathrm{m}$ from the top surface for (a), (b), (c) and (d), respectively. 
In the 15-20 micron region, a striped structure in the same direction as the director was observed.

The striped shape around the 15-20 micron region is supposed to be an alignment polymer layer because of the agreement with its rubbed direction and the depth region observed. The domain-like structure in the 5-15 micron region was observed everywhere in the film and the size ranged from a few to tens of microns. Since the light scattering was higher at such domain positions and also they were not dependent on the polarization direction of the illumination light, it is supposed that the domains were composed of the disordered region of molecules.

To investigate the domain-like structure and its ordering condition, the polarized Raman measurements were performed for the same sample. A typical Raman spectrum is shown in Fig. 2(a). Based on the assignments reported, ${ }^{18,19}$ most of the strong peaks, 1140, 1188, 1410, 1455, $1602 \mathrm{~cm}^{-1}$ were assigned to the aromatic $\mathrm{CH}$ ring mode. By measuring $I_{x}$ and $I_{y}\left(I_{x}, I_{y}\right.$ : the Raman intensities when excited and detected in $x$-polarized or $y$-polarized directions, respectively), denoting $x$ and $y$ as the parallel and perpendicular directions to the director axes, we evaluated the molecular anisotropy in the film by calculation, $\left|I_{x}-I_{y}\right| /\left(I_{x}+I_{y}\right)$, which is shown in Fig. 2(b). The images obtained by using the different peaks showed almost the same images (ESI, $\uparrow$ Fig. S2). We could find inhomogeneous distribution of anisotropic and isotropic regions with their sizes similar to the domain-like structures observed by SpeCRPM. The scan in the wider area with 120 microns square revealed that the structure

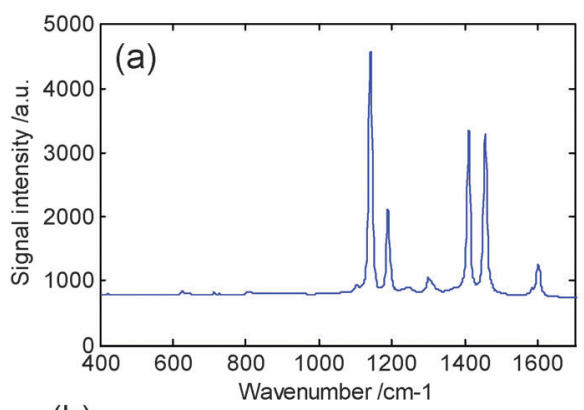

(b)

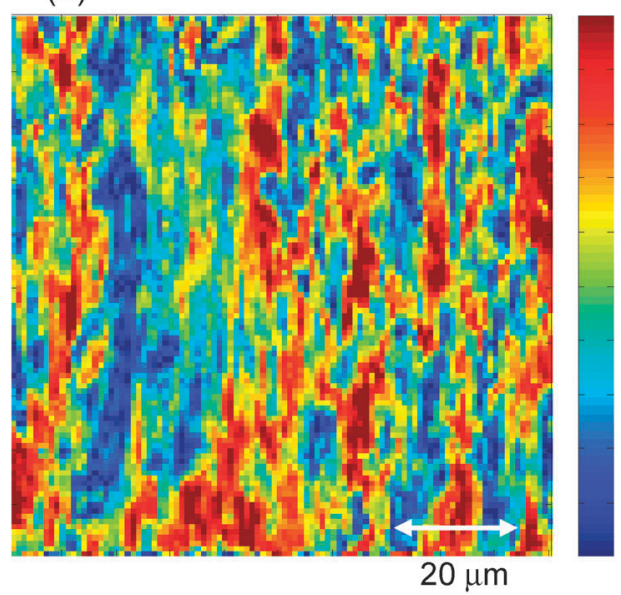

Fig. 2 (a) A Raman spectrum for a photomobile liquid crystalline polymer film. (b) Anisotropy imaging of the film using the peak at $1140 \mathrm{~cm}^{-1}$, with a scan step of $1 \mu \mathrm{m}$ with a field of view of $120 \times 120 \mu \mathrm{m}^{2}$. looked like a network structure aligned in the director axis. The isotropic regions correspond to the domain-like structure observed by SpecRPM. It is considered that the film has a network-like structure consisting of two different regions; isotropically and anisotropically aligned molecules, and that each region was likely to be aligned in the director axis.

Next, we observed the dynamic change in the images by UV irradiation at the depth position at 8.0 and $18.4 \mu \mathrm{m}$ from the surface. Hereinafter, the layers were called a domain layer and alignment layer, respectively. The UV light was irradiated for the initial $1 \mathrm{~min}$, and the subsequent change was observed for another $4 \mathrm{~min}$. The results are shown in Fig. 3. In both the domain and alignment layers, apparent change was observed when the image was observed under the polarization parallel with the director direction, while only a slight change was observed for the polarization in the perpendicular direction (compare with the images in ESI, $\dagger$ Fig. S3).

At the alignment layer, the image change was observed throughout the image 30 seconds after starting the UV irradiation, while the
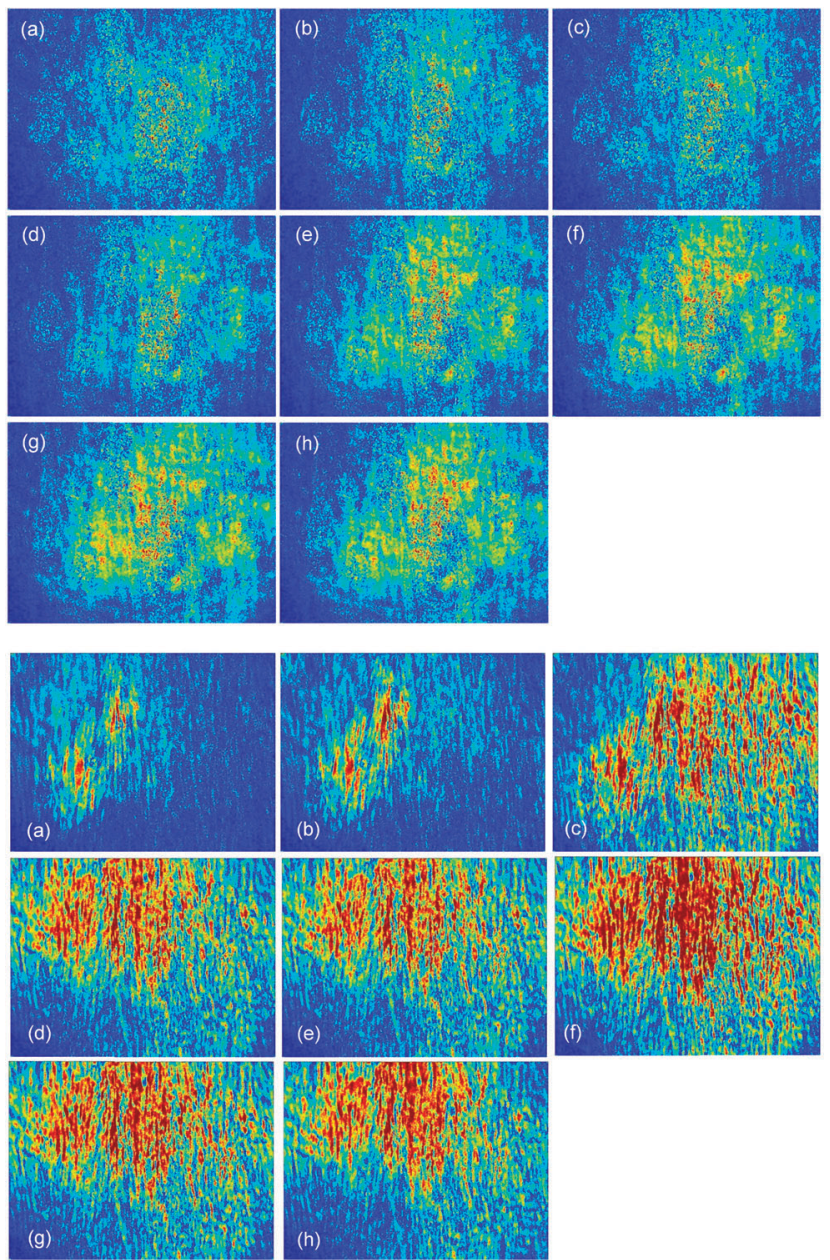

Fig. 3 Sequences of the images of a photomobile liquid crystalline polymer film by irradiation of UV light, and the above and bottom sequences correspond to the images at 8.0 and $18.4 \mu \mathrm{m}$ from the top surface. (a) - (h) Corresponds to the images, $0,15,30,45,60,90,120,180,240$ seconds after the UV irradiation was started, which was stopped after 60 seconds. 
change at the domain layer was observed 60 seconds after it. Looking at the domain layer carefully, the image change was mainly observed in the region except the domain-like islands. Furthermore we could confirm the shape of stripes after a few minutes, (Fig. 3 top (e)-(h)) which was a very similar structure as observed at the alignment layer (easily noticed by the videos in $\mathrm{ESI} \dagger)$.

The strong polarization dependence of the image change suggests that the signal enhancement was caused by the ordering of molecular alignment, not by the isotropic change such as the thermal expansion. Since it is known that the photoisomerization of azobenzene itself occurs very fast on the picosecond order, ${ }^{20,21}$ the image change was not caused by the structure change in azobenzene, and the alignment of the LC polymer network would be the origin of the image change, which would take tens of seconds, judging from the time lag for the image change after the UV irradiation. The delay of the image enhancement for the domain layer compared to that for the alignment layer indicates that the molecular ordering initially occurred at the alignment layer and then the ordering was travelled to the upper layer. This is supported by the fact that the structure observed after a few minutes showed a similar stripe pattern in the domain layer as observed in the alignment layer.

\section{Conclusions}

We studied the UV induced change in the photomobile LC polymer films using the microscopic techniques with a depth resolution of about $1 \mu \mathrm{m}$. The observed change at each slice of the depth suggested a network-like structure inside, which responded anisotropically to the UV light tens of seconds after the irradiation, and the change stayed a while on minute order. Since the rubbed surface at the alignment layer, which was the bottom of the layer, was gradually observed even in the middle of the film, it is suggested that the collaborative interaction travelled inside the film on the order of microns. This kind of collaborative molecular interactions is very important in the devices using LCs and polymers, and the microscopic observation with a depth resolution and with a high speed detection would give much insight of the collaborative interactions, which would be beneficial for the development of new devices.

\section{Notes and references}

1 R. Zentel, Adv. Mater., 1989, 1, 329.

2 S. M. Kelly, J. Mater. Chem., 1995, 5, 2047-2061.

3 H. Finkelmann, E. Nishikawa, G. G. Pereira and M. Warner, Phys. Rev. Lett., 2001, 87, 015501.

4 Y. Yu and T. Ikeda, J. Photochem. Photobiol., C, 2004, 5, 247-265. 5 T. Seki, Polym. J., 2004, 36, 435-454.

6 M. Yamada, M. Kondo, J. I. Mamiya, Y. L. Yu, M. Kinoshita, C. J. Barrett and T. Ikeda, Angew. Chem., Int. Ed., 2008, 47, 4986-4988.

7 M. Yamada, M. Kondo, R. Miyasato, Y. Naka, J.-i. Mamiya, M. Kinoshita, A. Shishido, Y. Yu, C. J. Barrett and T. Ikeda, J. Mater. Chem., 2009, 19, 60-62.

8 M. Yamada, M. Kondo, J.-I. Mamiya, Y. Yu, M. Kinoshita, C. J. Barrett and T. Ikeda, Angew. Chem., 2008, 120, 5064-5066.

9 J.-I. Mamiya, Polym. J., 2013, 45, 239-246.

10 T. Fujii, S. Kuwahara, K. Katayama, K. Takado, T. Ube and T. Ikeda, Phys. Chem. Chem. Phys., 2014, 16, 10485-10490.

11 M. Okuda and K. Katayama, Chem. Phys. Lett., 2007, 443, 158-162.

12 M. Okuda and K. Katayama, J. Phys. Chem. A, 2008, 112, 4545-4549.

13 T. Chiba, H. Inoue, S. Kuwahara and K. Katayama, J. Photochem. Photobiol., A, 2013, 266, 1-5.

14 M. Arai, T. Fujii, H. Inoue, S. Kuwahara and K. Katayama, Anal. Sci., 2013, 29, 401-404.

15 C. Youngwoong, P. Hosseini, W. Choi, R. R. Dasari, P. T. C. So and Z. Yaqoob, Opt. Lett., 2014, 39, 6062-6065.

16 J. W. Kang, N. Lue, C.-R. Kong, I. Barman, N. C. Dingari, S. J. Goldfless, J. C. Niles, R. R. Dasari and M. S. Feld, Biomed. Opt. Express, 2011, 2, 2484-2492.

17 J. W. Kang, F. T. Nguyen, N. Lue, R. R. Dasari and D. A. Heller, Nano Lett., 2012, 12, 6170-6174.

18 F. Lagugné Labarthet and C. Sourisseau, J. Raman Spectrosc., 1996, 27, 491-498.

19 F. L. Labarthet, J. L. Bruneel, T. Buffeteau and C. Sourisseau, J. Phys. Chem. B, 2004, 108, 6949-6960.

20 T. Fujino, S. Y. Arzhantsev and T. Tahara, J. Phys. Chem. A, 2001, 105, 8123-8129.

21 N. Tamai and H. Miyasaka, Chem. Rev., 2000, 100, 1875-1890. 